\title{
Chemotherapy and Biologic Therapy for Squamous Cell Cancers of the Head and Neck
}

\section{Sameer Mahesh}

Assistant Professor, Northeast Ohio College of Medicine, Jean B and Milton Cooper Cancer Center, Akron, OH

Correspondence: Sameer Mahesh, Assistant Professor, Northeast Ohio College of Medicine, Jean B and Milton Cooper Cancer Center, 161n Forge St. Suite 198, Akron, OH 44304, e-mail: maheshs@summa-health.org

\begin{abstract}
Squamous cell carcinoma of the head and neck presents a treatment challenge owing to the complexity of the anatomy of the head and neck region. Till recently, tobacco and alcohol were thought to be the main risk factors. Recently, human papilloma virus (HPV) has gained significant attention in the etiology of oropharyngeal cancer. Depending on the location of the tumor, surgery as a primary treatment can be quite debilitating due to cosmetic as well as functional reasons. Nonsurgical (radiotherapy or chemoradiotherapy) modalities are preferred as initial treatments of oropharynx, hypopharynx and larynx primaries. A multidisciplinary approach is recommended for treatment of these malignancies.
\end{abstract}

Keywords: Head and neck cancer, chemotherapy, cetuximab.

\section{INTRODUCTION}

Squamous cell carcinoma of the head and neck (SCCHN) is diagnosed in excess of half a million patients worldwide. It is the most common histologic subtype, accounting for 85 to $95 \%$ of cancers of the head and neck. The other anatomic sites-thyroid gland and salivary glands have cancers that are common histologic variants of adenocarcinoma and will not be included in this discussion. Chemotherapeutic agents that have activity against SCCHN include but are not restricted to platinum agents (cisplatin and carboplatin), fluorouracil (5-FU), docetaxel, paclitaxel, methotrexate, vinorelbine and bleomycin. Of these, the platinum agents, 5-FU and taxanes are most active and form the backbone of most chemotherapeutic regimens. Recently, biologic agents have also been shown to be active, the most widely used agent being Cetuximab which is a monoclonal antibody to the epidermal growth factor receptor (EGFR).

Chemotherapy use in SCCHN can be divided into the following categories which will be addressed in this review. Important studies are summarized in Table 1.

- Postoperative chemoradiotherapy (adjuvant treatment)

- Sequential chemoradiotherapy (induction chemotherapy followed by definitive local therapy)

- Concomitant chemoradiotherapy for organ preservation

- Concomitant chemoradiotherapy for unresectable disease

- Chemotherapy for recurrent and/or metastatic disease.
Biologic therapy (cetuximab) can be used in the following settings:

- Concomitant with radiotherapy for unresectable disease or organ preservation

- Recurrent/metastatic disease.

Issues in management of nasopharyngeal cancer are quite different which are addressed below. However, cisplatinbased concurrent chemoradiotherapy is essentially the standard for management of locally advanced nasopharyngeal cancer.

\section{POSTOPERATIVE CHEMORADIOTHERAPY (ADJUVANT TREATMENT)}

Two randomized trials clarified the role of postoperative chemoradiotherapy as adjuvant treatment. These studies conducted by European organization for research and treatment of cancer (EORTC) ${ }^{1}$ and radiation therapy oncology group (RTOG) ${ }^{2}$ addressed the question of whether the addition of cisplatin to standard postoperative radiotherapy based on pathologic criteria would improve the outcome of patients. The experimental arms of both studies consisted of standard fractionation radiation with concurrent cisplatin $\left(100 \mathrm{mg} / \mathrm{m}^{2}\right)$ on days 1,22 , and 43 . The 5-year results of the EORTC study indicated significant improvement in progression-free survival (47\% compared with $36 \%, \mathrm{p}=0.04)$, and overall survival (53\% compared 
with $40 \%, p=0.02$ ) in favor of concurrent cisplatin plus radiation. The findings of the RTOG study initially demonstrated a significant advantage with combinedmodality adjuvant therapy for the first two outcomes, but not for overall survival (3-year survival was $56 \%$ compared with $47 \% ; p=0.09$ ). In both studies, toxicity was greater with concurrent chemoradiotherapy. Although the treatment was very similar, the high-risk pathologic features were not uniform and the study populations differed. The entry criteria for the RTOG study were the presence of multiple positive nodes, extracapsular extension of tumor, or a positive margin. In contrast, the EORTC trial defined high risk as positive margin, extracapsular extension of nodal disease, vascular embolisms, or perineural disease; for oral cavity or oropharynx primary sites, high risk was defined as positive nodes at level IV or V. These differences may partly explain the variable outcome of the two trials. In an effort to reconcile these two trial results, a pooled analysis was performed that showed that the subsets of patients in both trials with significant benefit from cisplatin added to radiotherapy had either microscopically involved margins or extracapsular extension of disease in neck nodes. ${ }^{3}$ The presence of either or both of these risk factors is, therefore, considered a definite indication for adjuvant chemoradiotherapy.

\section{SEQUENTIAL CHEMORADIOTHERAPY (INDUCTION CHEMOTHERAPY FOLLOWED BY DEFINITIVE LOCAL THERAPY)}

In the 1980-2000 period there were multiple trials evaluating the role of induction chemotherapy followed by definitive local treatment. ${ }^{4-11}$ Chemotherapy regimens were mainly cisplatin based combinations with cisplatin and 5-FU dominating the literature in the 1990s. In spite of high antitumor activity of the induction chemotherapy, all but two trials did not show a benefit of induction chemotherapy for locoregional control or overall survival. In one of the randomized controlled trial, 237 patients with stages III and IV cancer of the oral cavity, oropharynx, hypopharynx, or paranasal sinus were randomly assigned to receive standard treatment or four cycles of cisplatin and 5-FU infusion followed by standard treatment. ${ }^{8}$ The trial included patients with resectable or unresectable disease. The control arm, or standard local-regional treatment, was prospectively determined for each patient based on their resectability status. There was no difference in overall survival or localregional control between the two groups, although there was a significant reduction in the incidence of distant metastases with the addition of chemotherapy. Of interest, on subset analysis, all outcomes improved for the patients with unresectable disease (3-year survival of $10 \%$ for those patients treated with radiation compared with $24 \%$ for those receiving induction chemotherapy; $\mathrm{p}=0.04$ ); only distant control improved for resectable disease with chemotherapy. In the other positive study conducted by the Groupe d'Etude des Tumeurs de la Tete Et du Cou (GETTEC), 318 patients with squamous cell carcinoma of the oropharynx in whom curative therapy was considered feasible, were randomized to three cycles of cisplatin and 5-FU followed by locoregional treatment or the same locoregional treatment alone. ${ }^{9}$ Median overall survival was 5.1 years in the chemotherapy arm vs 3.3 years in the control arm $(\mathrm{p}=0.03)$. Difference in event free survival was not statistically significant.

A meta-analysis of 63 randomized trials of local-regional treatment with or without chemotherapy was performed using updated patient data and was published in 2000. In this comprehensive review, there was no significant survival benefit from the addition of induction chemotherapy (31 trials; 5,269 patients; $\mathrm{HR}=0.95 ; 95 \% \mathrm{CI} ; 0.88,1.01 ; \mathrm{p}=$ $0.10)$. A subgroup analysis focusing on trials in which induction cisplatin and 5-FU was used showed a significant survival benefit for this regimen $(\mathrm{HR}=0.88 ; 95 \% \mathrm{CI} ; 0.79$, $0.97, \mathrm{p}=0.05) .{ }^{12}$ Taken collectively, induction chemotherapy was not strongly favored in standard clinical practice.

Recently published trials that incorporated the use of docetaxel have rekindled an interest in induction chemotherapy. The TAX 323 trial enrolled more than 300 patients with locally advanced unresectable disease. The control arm consisted of four cycles of cisplatin and 5-FU (PF) followed by radiotherapy, and the experimental arm consisted of four cycles of docetaxel, $75 \mathrm{mg} / \mathrm{m}^{2}$; cisplatin, $75 \mathrm{mg} / \mathrm{m}^{2} ; 5-\mathrm{FU}, 750 \mathrm{mg} / \mathrm{m}^{2}$ per day continuous infusion for 5 days (TPF) followed by radiotherapy. ${ }^{13}$ The response rate to induction of the three-drug regimen was significantly higher compared with that of the two-drug regimen (68\% vs $54 \%, \mathrm{p}=$ ?0.006) as was progression free survival (11 months vs 8.2 months, $\mathrm{p}=0.007)$ and overall survival $(18.8$ months $v s 14.5$ months, $\mathrm{p}=$ ?0.02). There was also less toxicity with the three-drug combination than with the twodrug combination. The TAX 324 trial randomly assigned 501 patients with unresectable or resectable disease (all sites) to three cycles of standard cisplatin and 5-FU or to combination docetaxel, cisplatin, and 5-FU (docetaxel, 75 
$\mathrm{mg} / \mathrm{m}^{2}$; cisplatin, $100 \mathrm{mg} / \mathrm{m}^{2} ; 5-\mathrm{FU}, 1,000 \mathrm{mg} / \mathrm{m}^{2}$ per day continuous infusion for 4 days). ${ }^{14}$ Definitive local therapy in both arms consisted of standard radiotherapy plus weekly carboplatin [area under the curve (AUC) $=1.5$ ]. The three drug regimen showed higher responses compared to the two drug regimen though not statistically significant $(72 \%$ $v s 64 \%, \mathrm{p}=0.07)$. Progression free survival (36 months vs 13 months, $\mathrm{p}=0.004)$, overall survival (71 months vs 30 months) and locoregional failure rate (30vs $38 \%, \mathrm{p}=0.04)$ were all in favor of the three drug regimen. Incidence of distant metastasis was similar in both arms.

Based on the above trials, when induction chemotherapy is considered, docetaxel, cisplatin and 5-FU should be the standard of care.

\section{CONCOMITANT CHEMORADIOTHERAPY FOR ORGAN PRESERVATION}

Organ preservation is particularly important for tumors of the oropharynx (preserve swallowing) and larynx/ hypopharynx (preserve voice). Initial studies conducted were based on induction chemotherapy for patients with resectable disease while subsequent studies focused on concurrent chemoradiotherapy. It was larynx preservation that set the tone for organ preservation. The department of veterans affairs laryngeal cancer study group (VALSCG) conducted a randomized study involving 332 patients with resectable T2-T4 laryngeal cancer. Patients were randomized to total laryngectomy followed by adjuvant radiation or to three cycles of induction cisplatin and 5-FU followed by radiation. Surgery was reserved for patients who had an inadequate response, persistent disease or relapse in the latter group. ${ }^{15}$ The larynx was preserved in $64 \%$ of patients overall and in $64 \%$ of long-term survivors. There were more local recurrences and fewer distant recurrences in the chemotherapy group. Overall survival was similar between the two groups. On multivariate analysis, T4 and N2 disease significantly predicted treatment failure with $56 \%$ of $\mathrm{T} 4$ lesions requiring laryngectomy eventually. A similar study was conducted by the EORTC with resectable T2-T4 hypopharynx cancer. Randomized patients received either primary surgery followed by radiation or three cycles of induction therapy with cisplatin and 5-FU followed by radiation. Of note, only complete responders after 2 or 3 cycles on induction chemotherapy received radiation. Nonresponders and partial responders received surgery. Locoregional failures were similar in the 2 arms, while distant recurrences were fewer in the induction chemotherapy arm as compared to the primary surgery arm (25\% vs $36 \%)$. Although median survivals were 44 months in the induction chemotherapy arm and 25 months in the immediate surgery arm, these were statistically not significant. More importantly, at five years, $35 \%$ of patients in the induction chemotherapy arm had an intact larynx. These two studies, established induction chemotherapy followed by radiation as an organ preservation strategy.

Subsequently, interest in concurrent chemoradiotherapy arose, and in fact the Intercrop 91-11 study randomized 547 patients with T2-low-volume T4, nonmetastatic squamous cell cancers of the larynx to radiotherapy alone, radiotherapy with concomitant cisplatin administered on days 1, 22 and 43, or induction cisplatin and 5-FU, followed by radiotherapy for patients having a partial or complete response to induction chemotherapy at the primary site. ${ }^{16}$ Neck dissection was performed for all patients initially presenting with $\mathrm{N} 2$ or N3 disease. Laryngectomy was performed for patients with insufficient response, persistent disease or local recurrence. Two year larynx preservation rates were $88 \%$ in the concomitant chemoradiotherapy arm compared with $75 \%$ in the induction arm $(\mathrm{p}=0.005)$ and $70 \%$ in the radiotherapy alone arm $(\mathrm{p}<0.001)$. Locoregional control was also superior in the concomitant arm $(78 \%$ vs $61 \%$ vs $56 \%$ respectively). Distant metastasis free survivals were superior the chemotherapy arms as compared to radiation alone arm (61\% vs 52\% vs 44\%). Overall survival was similar in all three groups.

For the oropharynx tumors, the Group d'Oncologie Radiotherapie Tete et Cou (GORTEC) randomized 226 patients with stage III or IV squamous cell cancer of the oropharynx to radiotherapy alone, or radiotherapy with concomitant carboplatin $\left(70 \mathrm{mg} / \mathrm{m}^{2}\right)$ and $5-\mathrm{FU}\left(600 \mathrm{mg} / \mathrm{m}^{2}\right.$ daily continuous infusion for 4 days) starting on days 1,22 and 43 of radiation. ${ }^{17}$ Three-year disease free survival ( $42 \%$ vs 20\%) and overall survival (51\% vs 31\%) were superior in the combined modality arm. Mucositis, requirement of feeding tube, treatment related weight loss and myelosuppression was greater in the combined arm.

\section{CONCOMITANT CHEMORADIOTHERAPY FOR UNRESECTABLE DISEASE}

Multiple studies were published from 1990-2000, addressing the role of chemotherapy in addition to radiotherapy for unresectable SCCHN. ${ }^{18-21}$ In 2003, Adelstein et al published the results of an Intergroup trial that led to the current standard of care. ${ }^{22}$ In this study, 295 patients were 
randomized to a radiotherapy alone arm, radiotherapy with cisplatin $100 \mathrm{mg} / \mathrm{m}^{2}$ on days 1,22 and 43 , or split dose radiotherapy with cisplatin and 5-FU. The 3-year overall survivals were $23 \%, 37 \%$ and $27 \%$ respectively. Only the difference between the first 2 arms was significant ( $\mathrm{p}=$ $0.014)$ establishing radiation therapy with high dose cisplatin as the current standard of care. To address if hyperfractionated radiotherapy would overcome the advantage of concurrent cisplatin, a phase 3 trial randomized 130 patients with stage III and IV SCCHN to hyperfractionated radiotherapy with or without concurrent cisplatin given at $6 \mathrm{mg} / \mathrm{m}^{2}$ daily. Five-year overall survival was in favor of the combined modality group (46\% vs 25\%, $\mathrm{p}=0.075) .{ }^{23} \mathrm{~A}$ meta-analysis also showed a survival advantage of combined modality treatment as compared to radiation alone. There was an $8 \%$ advantage in survival at 5 years in spite of significant heterogeneity of trials. ${ }^{12}$ Based on the above data, radiotherapy with cisplatin at $100 \mathrm{mg} / \mathrm{m}^{2}$ on days 1, 22 and 43 of radiation should be the standard of care for patients with SCCHN. The role of biologic therapy in this setting is addressed below.

\section{CHEMOTHERAPY FOR INCURABLE RECURRENT/METASTATIC DISEASE}

Chemotherapy for incurable recurrent or metastatic disease is palliative and is usually platinum based. Cisplatin or carboplatin, with or without 5-FU is currently the standard regimen for this subgroup. The benefit obtained from addition of 5-FU is modest at best. In a phase III trial, 249 patients with recurrent head and neck cancers were randomized to cisplatin alone, 5FU alone or the combination. Although the combination arm had increased response rates, there was no increase in overall survival in the combination arm. ${ }^{24}$ On subgroup analysis, OS benefit was limited to patients with good performance status or poorly differentiated histology. Combination chemotherapy should be reserved for good performance status patients who might be in a better position to benefit from the increased response rates.

The addition of taxanes to the armamentarium of drugs was anticipated to improve the outcome in the subgroup. Unfortunately, this was not the case. In a phase III trial, patients with metastatic, recurrent or locally advanced head and neck cancers were randomized to cisplatin and 5-FU or cisplatin and taxol. There was no difference in response rates (27\% vs 26\%) or OS (8.7 months vs 8.1 months).

The benefit of adding the anti-EFGR monoclonal antibody cetuximab was recently shown to improved outcomes in this group of patients and is addressed below. ${ }^{25}$

\section{WHERE DO WE STAND NOW? CONCURRENT CHEMORADIOTHERAPY OR INDUCTION THERAPY FOLLOWED BY DEFINITIVE LOCAL THERAPY?}

The data incorporating docetaxel in the induction regimen is fairly recent. ${ }^{13,14}$ Concurrent cisplatin-based chemoradiotherapy has a long track record in terms of improving locoregional control and in fact is superior to $\mathrm{CF}$ based induction chemotherapy for the same. ${ }^{16}$ However, TPF is superior to CF for locoregional control. ${ }^{14}$ Induction chemotherapy has superior outcomes in terms of reducing distant metastasis while that of concurrent treatment is at best questionable. ${ }^{8}$ Induction therapy runs the danger of causing toxicities to the patient thereby delaying definitive treatment. The debate between these two approaches exists since there is no comparative trial that has data mature

TABLE 1: Phase 3 studies in previously untreated SCCHN

\begin{tabular}{|c|c|c|c|c|c|}
\hline Study & $\begin{array}{l}\text { No. of } \\
\text { patients }\end{array}$ & $\begin{array}{l}\text { Study } \\
\text { description }\end{array}$ & Population & $\begin{array}{l}\text { Median PFS } \\
\text { (months) }\end{array}$ & $\begin{array}{l}\text { Median OS } \\
\text { (months) }\end{array}$ \\
\hline EORTC $22931^{1}$ & 334 & Cisplatin + RT vs RT alone & Postoperative & 55 vs 23 & 72 vs 32 \\
\hline RTOG $9501^{2}$ & 459 & Cisplatin + RT vs RT alone & Postoperative & Not reported & 44.9 vs 31.9 \\
\hline RTOG $9111^{16}$ & 547 & $\begin{array}{l}\text { Cisplatin + RT vs Cisplatin } \\
+5-\mathrm{FU} \rightarrow \mathrm{RT} \text { vs RT alone }\end{array}$ & Larynx Ca & 47 vs 45 vs $34^{+}$ & 55 vs 59 vs 54 \\
\hline $\operatorname{TAX} 323^{13}$ & 358 & $\mathrm{TPF} \rightarrow \mathrm{RT} v s \mathrm{PF} \rightarrow \mathrm{RT}$ & Unresectable & 11.0 vs 8.2 & 18.8 vs 14.5 \\
\hline TAX $324^{14}$ & 501 & $\begin{array}{l}\text { TPF } \rightarrow \text { carboplatin + RT vs } \\
\mathrm{PF} \rightarrow \text { carboplatin }+\mathrm{RT}\end{array}$ & $\begin{array}{l}\text { Resectable and } \\
\text { unresectable }\end{array}$ & 36 vs 13 & 70 vs 31 \\
\hline Intergroup ${ }^{22}$ & 295 & $\begin{array}{l}\text { Cisplatin + RT vs cisplatin } \\
+5-\mathrm{FU}+\text { split dose RT vs RT }\end{array}$ & Unresectable & Not reported & $\begin{array}{l}19.1 \text { vs } 13.8 \\
\text { vs } 12.6\end{array}$ \\
\hline Bonner et $\mathrm{al}^{34}$ & 424 & Cetuximab + RT vs RT & $\begin{array}{l}\text { Resectable and } \\
\text { unresectable }\end{array}$ & 17.1 vs 12.4 & 49.0 vs 29.3 \\
\hline
\end{tabular}

RT denotes radiotherapy, TPF docetaxel, cisplatin and 5-FU, PF cisplatin + 5-FU.

+ Primary endpoint of the trial was larynx preservation rate. Results in this trial are reported as laryngectomy-free survival. 
enough to state the advantage of one over the other. Each case should be evaluated on an individual basis by a multidisciplinary team in terms of addressing the risks of local and distant disease. It is also important for the multidisciplinary team to be involved in the care of these patients right from the start so that induction therapy can be aborted and definitive treatment started at the earliest in case of toxicities. Off protocol, it is reasonable to use induction chemotherapy among patients who need therapy immediately. It is also reasonable to use it in patients with bulky primaries (T4 disease) and advanced nodal presentations (N2b, N2c, and N3 disease).

Preliminary data comparing these two approaches in patients with unresectable disease has been presented. ${ }^{26} \mathrm{In}$ this phase III randomized study, 439 patients with locally advanced unresectable SCCHN were randomized to three cycles of induction chemotherapy (PF or TPF) followed by standard dose radiotherapy with concurrent cisplatin at $100 \mathrm{mg} / \mathrm{m}^{2}$ on days 1,22 and 43 (arm 1) or chemoradiotherapy alone (arm 2). ${ }^{26}$ Median time to treatment failure was 12.5 months in arm 1 vs 4.9 months in arm 2. Locoregional control rates were $61 \%$ and $44.5 \%$ respectively. Grade 3 and 4 toxicities were higher in arm A. Febrile neutropenia rates were $10 \%$ and $1 \%$ respectively. Of note, overall survival was 37 months and 27 months but not statistically significant (HR $0.85 ; 0.63-1.15$ ). Final publication is awaited.

\section{BIOLOGIC THERAPY}

Epidermal growth factor receptor (EGFR) is expressed in virtually all SCCHN and its presence is associated with a worse prognosis. ${ }^{27-29}$ EGFR is a member of the Erb-B family of receptors that are activated by numerous ligands. Activation leads to dimerization of the receptors which is followed by a series of changes that eventually leads to activation of transcription factors that regulate gene expression involved in cell migration, adhesion, proliferation, angiogenesis and inhibition of apoptosis (Fig. 1). ${ }^{30}$ Targeting EGFR seems a logical way of improving outcomes in SCCHN. Cetuximab is an IgG1 monoclonal antibody that inhibits ligand binding to EGFR and also promotes antibody dependant cell-mediated cytotoxicity. ${ }^{31-33}$

In a landmark trial, 424 patients with locoregionally advanced SCCHN were randomized to cetuximab plus radiotherapy or radiotherapy alone. Cetuximab was given at $400 \mathrm{mg} / \mathrm{m}^{2}$ as initial dose followed by $250 \mathrm{mg} / \mathrm{m}^{2}$ every

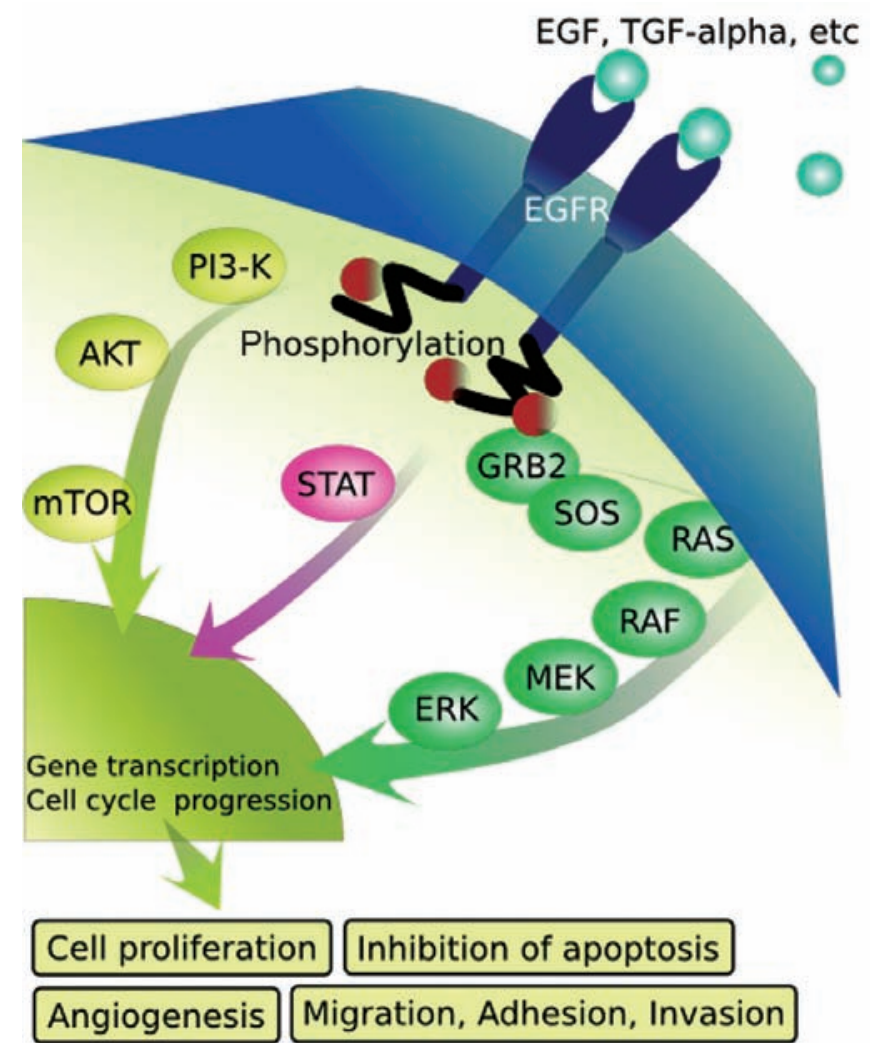

FIGURE 1: EGFR Signaling Pathway: A simplified version showing signaling cascades after dimerization of the EGFR. Dimerization leads to activation of the PI3-AKT, Ras/MAPK (mitogen associated protein kinase), and JAK (Janus Kinase)-STAT pathways. These eventually lead to activation of numerous transcription factors that regulate gene expression that support cell proliferation, angiogenesis, inhibition of apoptosis and invasion. EGF denotes epidermal growth factor, TGFalpha transforming growth factor alpha, PI3-K phosphatidylinositol 3-kinase, AKT protein kinase B, mTOR mammalian target of rapamycin, STAT signal transducers and activators of transcription, GRB2 growth factor receptor-bound protein 2, SOS son of sevenless, MEK mitogen activated protein kinase, ERK extracellular signalregulated kinase

week. ${ }^{34}$ Median duration of locoregional control was 24.4 months in the combined treatment arm as compared to 14.9 months in the radiotherapy alone arm. Overall survivals were 49 months and 29.3 months respectively. Grade 3 or higher toxic effects that were higher in the cetuximab arm were acneiform rash and infusion reactions. Other toxicities including mucositis were similar. Of note grade 3-4 radiation dermatitis rates were $23 \%$ in the combined arm and $18 \%$ in the radiation alone arm. Radiation dermatitis which is classically within the radiation fields should be distinguished from the characteristic acneiform rash (Fig. 2). A recent survey of the EORTC institutes showed that the rate of severe radiation dermatitis was approximately $49 \%$ in patients treated concurrently with cetuximab and radiation. ${ }^{35}$ The management of radiation dermatitis with or without 


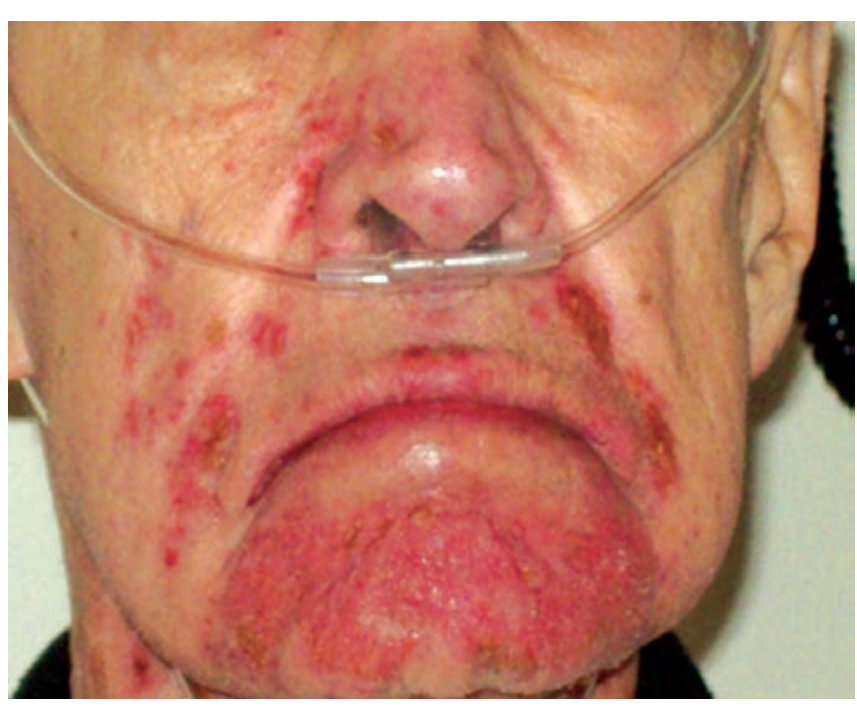

FIGURE 2: Dermatitis during week 3 in a patient receiving concurrent radiotherapy and cetuximab. The rash got significantly worse the subsequent week in spite of supportive treatment as outlined by the guidelines. Cetuximab was held for one dose and radiotherapy for three days. The rash responded dramatically to topical bacitracin ointment. The patient subsequently completed treatment with no further delays

cetuximab is addressed elsewhere. ${ }^{36}$ Of note, there was no chemotherapy arm in this study so it is impossible to make a comparison between cisplatin-based radiation and cetuximab-based radiation. RTOG 0522 is a randomized phase 3 study comparing accelerated radiotherapy and cisplatin with or without cetuximab. It has closed to accrual and we will have to wait a few years before any efficacy data is available. Till then, cetuximab with radiotherapy should be considered for patients with locally advanced SCCHN who are unfit for cisplatin-based chemotherapy. It should not be used in addition to cisplatin and radiotherapy off study.

Cetuximab has also shown activity in the advanced disease setting (Table 2). In a randomized phase III study, 442 patients with previously untreated recurrent or metastatic disease were randomized to cisplatin $100 \mathrm{mg} / \mathrm{m}^{2}$ or carboplatin (area under the curve of 5 milligram/milliliter/ per minute), 5 -FU (1000 $\mathrm{mg} / \mathrm{m}^{2}$ continuous infusion per day for 4 days) every 3 weeks for 6 cycles, with or without cetuximab $\left(400 \mathrm{mg} / \mathrm{m}^{2}\right.$ initial dose then weekly at $250 \mathrm{mg} /$ $\left.\mathrm{m}^{2}\right) .{ }^{25}$ The addition of cetuximab led to an improvement in response rates from 20 to $36 \%$, prolonged median PFS from 3.3 to 5.6 months and median OS from 7.4 to 10.1 months. Grade 3 and 4 toxicities that were greater in the cetuximab arm included sepsis (4\% vs <1\%), rash (9\% vs $<1 \%$ ) and infusion reactions (3\% vs 0\%). ECOG 5397 was a study that randomized patients 117 patients to cisplatin in combination with cetuximab or cisplatin alone. ${ }^{37}$ Although the response rates were increased in the combination arm (26\% vs 10\%), this did not translate into an improvement in overall survival (9.2 vs 8.0 months, $\mathrm{p}=0.21)$. In another study, patients with disease progression on two to six cycles of platinum therapy received single-agent cetuximab (initial dose $400 \mathrm{mg} / \mathrm{m}^{2}$ followed by subsequent weekly doses of $250 \mathrm{mg} / \mathrm{m}^{2}$ ). Response rate was $13 \%$ and median time to progression was 70 days ${ }^{38}$ Cetuximab is currently approved in combination with radiotherapy for locally advanced SCCHN and in patients with recurrent or metastatic disease progressing after platinum based chemotherapy. Patients should be monitored for serum magnesium periodically during treatment since hypomagnesemia is a known complication of cetuximab.

Erlotinib and gefitinib are oral tyrosine kinase inhibitors that act by inhibiting the phosphorylation of EGFR intracellularly. They have only modest clinical activity in SCCHN when used as single agents. ${ }^{39,40}$ A three arm study compared oral gefitinib at $250 \mathrm{mg} /$ day or $500 \mathrm{mg}$ /day $v s$ methotrexate $40 \mathrm{mg} / \mathrm{m}^{2}$ weekly intravenously. There was no difference in survival between the three arms (median survivals were 5.6 months, 6.0 months and 6.7 months respectively). Of note, there was an increase in tumorhemorrhage type events in the gefitinib groups $(8.9 \%$,

TABLE 2: Studies involving cetuximab in recurrent or metastatic SCCHN

\begin{tabular}{lcllll}
\hline Study & $\begin{array}{c}\text { No. of } \\
\text { patients }\end{array}$ & Treatment & Population & Response Rate (\%) & Survival (mo) \\
\hline $\begin{array}{l}\text { Bonner et al }\left.\right|^{34} \\
\text { EXTREME }^{25}\end{array}$ & 442 & $\begin{array}{l}\text { Platinum + 5-FU + cetuximab vs } \\
\text { platinum + 5-FU }\end{array}$ & First-line recurrent & 35.6 vs 19.5 & 10.1 vs 7.4 \\
ECOG $5397^{37}$ & 117 & $\begin{array}{l}\text { Cisplatin + cetuximab vs cisplatin } \\
\text { Cetuximab }\end{array}$ & $\begin{array}{l}\text { First-line recurrent } \\
\text { Platinum resistant }\end{array}$ & $\begin{array}{l}26 \text { vs } 10 \\
13\end{array}$ & 9.2 vs $8.0^{+}$ \\
\end{tabular}

EXTREME denotes Erbitux (cetuximab) in first line treatment of recurrent or metastatic head and neck cancer, ECOG-Eastern cooperative oncology group.

+ Value was not significant. 
gefitinib $250 \mathrm{mg} /$ day, $11.7 \%$, gefitinib $500 \mathrm{mg} /$ day, and $1.7 \%$, methotrexate). ${ }^{41}$ Studies are underway of combining these agents with chemotherapy in the first line setting in advanced disease.

\section{NASOPHARYNGEAL CANCER (NPC)}

NPC arises at a site that is clearly technically difficult to operate. It is unique in terms of etiology in the endemic areas (Southern China and Northern Africa) where it is related to Epstein-Barr virus in almost all cases. There are 3 subtypes, WHO type 1 (squamous cell cancer), WHO type 2 (nonkeratinizing cancer) and WHO type 3 (undifferentiated cancer). WHO types 2 and 3 are the predominant subtypes in endemic areas. It frequently presents with locoregional advanced disease and in fact should be considered when squamous cell cancer is found in level II or V lymph nodes in absence of a primary tumor.

Radiation therapy used to be the standard of care for locally advanced disease until the results of a large Intergroup study were published. Almost 200 patients with stage III and IV NPC were randomized to radiotherapy to a total dose of 70Gy or radiotherapy along with cisplatin $100 \mathrm{mg} /$ $\mathrm{m}^{2}$ every 21 days for 3 cycles concomitantly followed by three cycles of cisplatin $\left(80 \mathrm{mg} / \mathrm{m}^{2}\right)$ and 5-FU $(1000 \mathrm{mg} /$ $\mathrm{m}^{2} /$ day for 4 days). ${ }^{42}$ Approximately $90 \%$ of patients had stage 4 disease, and WHO subtype 1 was the commonest histology. The combined modality arm was superior to the radiation alone arm in terms of 5-year progression-free survival $(58 \%$ vs $29 \%, \mathrm{p}<0.001)$, and overall survival (67\% vs 37\%). Of note, approximately two-third of patients received all three doses of concurrent cisplatin and half received all three cycles of adjuvant cisplatin and 5-FU). Two other studies conducted in endemic areas have shown a significant survival advantage with concurrent platinum chemotherapy and radiation over radiation alone. ${ }^{43,44}$ These results have also been confirmed in two meta-analysis. ${ }^{45,46}$

Induction chemotherapy followed by radiotherapy as compared to radiotherapy alone have not been shown to improve overall though there have been mixed results in terms of progression-free survival. ${ }^{47-49} \mathrm{~A}$ recent randomized phase 2 study showed a survival advantage for induction chemotherapy with cisplatin and taxotere for 2 cycles followed by chemoradiotherapy with weekly cisplatin as compared to the same chemoradiotherapy arm alone. The 3 -year progression free survival for the neoadjuvant vs control arm was $88 \%$ and $59 \%$ while overall survival was 94\% and 68\% respectively. Induction chemotherapy for
NPC is not recommended out of a protocol setting. Taking into account the data above, radiation with concurrent cisplatin at $100 \mathrm{mg} / \mathrm{m}^{2}$ on days 1,22 and 43 followed by 3 cycles of cisplatin $\left(80 \mathrm{mg} / \mathrm{m}^{2}\right)$ and 5 -FU $\left(1000 \mathrm{mg} / \mathrm{m}^{2} /\right.$ day for 4 days) should be the standard of care for patients with stage IIB-IV NPC.

\section{FUTURE DIRECTIONS}

Management of SCCHN has evolved over the last few decades. Several advances have been made in terms of chemotherapeutic drugs, radiotherapy techniques and management of toxicities. However, novel approaches are required to improve the outcomes that platinum-based regimens have achieved. Incorporation of cetuximab is an important step in that direction. The results of RTOG 0522 are eagerly awaited. Meanwhile, the field of oncology has seen an explosion in the field of targeted therapy. Several of these agents in addition are being studied in phase 1 and 2 trials. Some of these agents are bevacizumab, sunitinib and cedarinib of which bevacizumab and sunitinib are already approved for other malignancies. If results are encouraging, it would be exciting to bring these drugs into frontline management.

\section{KEY POINTS}

- Concurrent cisplatin and radiation is the standard of care for adjuvant treatment of tumors that have positive microscopic margin or extracapsular extension

- If induction chemotherapy is planned, the preferred regimen is taxotere, cisplatin and 5-FU (TPF)

- Debate between induction therapy with TPF followed by local treatment $v s$ concurrent chemoradiation needs to be resolved. At this point, every case should be managed on an individualized basis

- Organ preserving strategies should be sought for tumors of the larynx, hypopharynx and oropharynx. Surgery should be used only in the salvage setting

- For unresectable SCCHN radiation with high dose cisplatin is the current standard of care

- Monoclonal antibody therapy with cetuximab in combination with radiation is an appropriate option for patients who need concurrent treatment but cannot tolerate chemotherapy.

- Results of cetuximab in addition to chemotherapy and radiation are eagerly awaited. Till then, cetuximab should not be combined with cisplatin and radiation in a nonprotocol setting 
- Additional studies using oral EGFR inhibitors and vascular endothelial growth factor inhibitors in advanced disease are eagerly awaited

- Nasopharyngeal cancer frequently presents with stage III and IV disease. Cisplatin and radiation given concurrently followed by three cycles of cisplatin and 5FU is the current standard of care for these patients.

\section{REFERENCES}

1. Bernier J, et al. Postoperative irradiation with or without concomitant chemotherapy for locally advanced head and neck cancer. N Engl J Med 2004;350(19):1945-52.

2. Cooper JS, et al. Postoperative concurrent radiotherapy and chemotherapy for high-risk squalors-cell carcinoma of the head and neck. N Engl J Med 2004;350(19):1937-44.

3. Bernier J, et al. Defining risk levels in locally advanced head and neck cancers: A comparative analysis of concurrent postoperative radiation plus chemotherapy trials of the EORTC (\#22931) and RTOG (\# 9501). Head Neck 2005;27(10):843-50.

4. Jota A, et al. A randomized EORTC study on the effect of preoperative polychemotherapy in pyriform sinus carcinoma treated by pharyngolaryngectomy and irradiation. Results from 5 to 10 years. Acta Chir Belg 1990;90(3):115-22.

5. Jaulerry C, et al. Induction chemotherapy in advanced head and neck tumors: Results of two randomized trials. Int J Radiat Oncol Biol Phys 1992;23(3):483-89.

6. Mazeron JJ, et al. Induction chemotherapy in head and neck cancer: Results of a phase III trial. Head Neck 1992;14(2):8591.

7. Depondt J, et al. Neoadjuvant chemotherapy with carboplatin/ 5-fluorouracil in head and neck cancer. Oncology 1993;(50 Suppl)2:23-27.

8. Paccagnella A, et al. Phase III trial of initial chemotherapy in stage III or IV head and neck cancers: A study by the Gruppo di Studio sui Tumori della Testa e del Collo. J Natl Cancer Inst, 1994;86(4):265-72.

9. Domenge $\mathrm{C}$, et al. Randomized trial of neoadjuvant chemotherapy in oropharyngeal carcinoma. French Groupe d'Etude des Tumeurs de la Tete et du Cou (GETTEC). Br J Cancer 2000;83(12):1594-98.

10. Licitra L, et al. Primary chemotherapy in resectable oral cavity squamous cell cancer: A randomized controlled trial. J Clin Oncol, 2003;21(2):327-33.

11. Lefebvre JL, et al. Larynx preservation in pyriform sinus cancer: Preliminary results of a European Organization for Research and Treatment of Cancer phase III trial. EORTC Head and Neck Cancer Cooperative Group. J Natl Cancer Inst 1996;88(13):89099.

12. Pignon JP, et al. Chemotherapy added to locoregional treatment for head and neck squamous-cell carcinoma: Three meta-analyses of updated individual data. MACH-NC Collaborative Group. Meta-Analysis of Chemotherapy on Head and Neck Cancer. Lancet 2000;355(9208):949-55.

13. Vermorken JB, et al. Cisplatin, fluorouracil, and docetaxel in unresectable head and neck cancer. N Engl J Med 2007;357(17):1695-704.
14. Posner MR, et al. Cisplatin and fluorouracil alone or with docetaxel in head and neck cancer. N Engl J Med 2007;357(17):1705-15.

15. Induction chemotherapy plus radiation compared with surgery plus radiation in patients with advanced laryngeal cancer. The Department of Veterans Affairs Laryngeal Cancer Study Group. N Engl J Med 1991;324(24):1685-90.

16. Forastiere AA, et al. Concurrent chemotherapy and radiotherapy for organ preservation in advanced laryngeal cancer. N Engl J Med 2003;349(22):2091-98.

17. Calais $\mathrm{G}$, et al. Randomized trial of radiation therapy vs concomitant chemotherapy and radiation therapy for advancedstage oropharynx carcinoma. J Natl Cancer Inst 1999;91(24): 2081-86.

18. Merlano M, et al. Five-year update of a randomized trial of alternating radiotherapy and chemotherapy compared with radiotherapy alone in treatment of unresectable squamous cell carcinoma of the head and neck. J Natl Cancer Inst 1996;88(9):583-89.

19. Brizel DM, et al. Hyperfractionated irradiation with or without concurrent chemotherapy for locally advanced head and neck cancer. N Engl J Med 1998;338(25):1798-804.

20. Wendt TG, et al. Simultaneous radiochemotherapy $v s$ radiotherapy alone in advanced head and neck cancer: A randomized multicenter study. J Clin Oncol 1998;16(4):1318-24.

21. Adelstein DJ, et al. Mature results of a phase III randomized trial comparing concurrent chemoradiotherapy with radiation therapy alone in patients with stage III and IV squamous cell carcinoma of the head and neck. Cancer 2000;88(4):876-83.

22. Adelstein DJ, et al. An intergroup phase III comparison of standard radiation therapy and two schedules of concurrent chemoradiotherapy in patients with unresectable squamous cell head and neck cancer. J Clin Oncol 2003;21(1):92-98.

23. Jeremic B, et al. Hyperfractionated radiation therapy with or without concurrent low-dose daily cisplatin in locally advanced squamous cell carcinoma of the head and neck: A prospective randomized trial. J Clin Oncol 2000;18(7):1458-64.

24. Jacobs $\mathrm{C}$, et al. A phase III randomized study comparing cisplatin and fluorouracil as single agents and in combination for advanced squamous cell carcinoma of the head and neck. J Clin Oncol 1992;10(2):257-63.

25. Vermorken JB, et al. Platinum-based chemotherapy plus cetuximab in head and neck cancer. N Engl J Med 2008;359(11):1116-27.

26. Hitt R. Final results of a randomized phase III trial comparing induction chemotherapy with cisplatin/5-FU or docetaxel/ cisplatin/5-FU follow by chemoradiotherapy (CRT) vs CRT alone as first-line treatment of unresectable locally advanced head and neck cancer (LAHNC). Journal of Clinical Oncology 2009;27(15s):Abstract 6009.

27. Rubin Grandis J, et al. Levels of TGF-alpha and EGFR protein in head and neck squamous cell carcinoma and patient survival. J Natl Cancer Inst 1998;90(11):824-32.

28. Chung $\mathrm{CH}$, et al. Increased epidermal growth factor receptor gene copy number is associated with poor prognosis in head and neck squamous cell carcinomas. J Clin Oncol 2006;24(25):417076.

29. Temam S, et al. Epidermal growth factor receptor copy number alterations correlate with poor clinical outcome in patients with head and neck squamous cancer. J Clin Oncol 2007;25(16):216470. 
30. Oda K, et al. A comprehensive pathway map of epidermal growth factor receptor signaling. Mol Syst Biol 2005;1:2005 0010.

31. Goldstein NI, et al. Biological efficacy of a chimeric antibody to the epidermal growth factor receptor in a human tumor xenograft model. Clin Cancer Res 1995;1(11):1311-18.

32. Li S, et al. Structural basis for inhibition of the epidermal growth factor receptor by cetuximab. Cancer Cell 2005;7(4):301-11.

33. Kimura H, et al. Antibody-dependent cellular cytotoxicity of cetuximab against tumor cells with wild-type or mutant epidermal growth factor receptor. Cancer Sci 2007;98(8):1275-80.

34. Bonner JA, et al. Radiotherapy plus cetuximab for squamouscell carcinoma of the head and neck. N Engl J Med 2006;354(6):567-78.

35. Giro $\mathrm{C}$, et al. High rate of severe radiation dermatitis during radiation therapy with concurrent cetuximab in head and neck cancer: Results of a survey in EORTC institutes. Radiother Oncol 2009;90(2):166-71.

36. Bernier J, et al. Consensus guidelines for the management of radiation dermatitis and coexisting acne-like rash in patients receiving radiotherapy plus EGFR inhibitors for the treatment of squamous cell carcinoma of the head and neck. Ann Oncol 2008;19(1):142-49.

37. Burtness B, et al. Phase III randomized trial of cisplatin plus placebo compared with cisplatin plus cetuximab in metastatic/ recurrent head and neck cancer: An Eastern Cooperative Oncology Group study. J Clin Oncol 2005;23(34):8646-54.

38. Vermorken JB, et al. Open-label, uncontrolled, multicenter phase II study to evaluate the efficacy and toxicity of cetuximab as a single agent in patients with recurrent and/or metastatic squamous cell carcinoma of the head and neck who failed to respond to platinum-based therapy. J Clin Oncol 2007;25(16):2171-77.

39. Cohen EE, et al. Phase II trial of ZD1839 in recurrent or metastatic squamous cell carcinoma of the head and neck. J Clin Oncol 2003;21(10):1980-87.

40. Soulieres D, et al. Multicenter phase II study of erlotinib, an oral epidermal growth factor receptor tyrosine kinase inhibitor, in patients with recurrent or metastatic squamous cell cancer of the head and neck. J Clin Oncol 2004;22(1):77-85.

41. Stewart JS, et al. Phase III study of gefitinib 250 compared with intravenous methotrexate for recurrent squamous cell carcinoma of the head and neck. J Clin Oncol 2009;27(11):1864-71.

42. Al-Sarraf $\mathrm{M}$, et al. Chemoradiotherapy vs radiotherapy in patients with advanced nasopharyngeal cancer: Phase III randomized Intergroup study 0099. J Clin Oncol 1998;16(4):1310-17.

43. Chan AT, et al. Overall survival after concurrent cisplatinradiotherapy compared with radiotherapy alone in locoregionally advanced nasopharyngeal carcinoma. J Natl Cancer Inst 2005;97(7):536-39.

44. Lin JC, et al. Phase III study of concurrent chemoradiotherapy $v s$ radiotherapy alone for advanced nasopharyngeal carcinoma: Positive effect on overall and progression-free survival. J Clin Oncol 2003;21(4):631-37.

45. Huncharek M, B Kupelnick. Combined chemoradiation vs radiation therapy alone in locally advanced nasopharyngeal carcinoma: Results of a meta-analysis of 1,528 patients from six randomized trials. Am J Clin Oncol 2002;25(3):219-23.

46. Baujat B, et al. Chemotherapy in locally advanced nasopharyngeal carcinoma: An individual patient data metaanalysis of eight randomized trials and 1753 patients. Int J Radiat Oncol Biol Phys 2006;64(1):47-56.

47. Chan AT, et al. A prospective randomized study of chemotherapy adjunctive to definitive radiotherapy in advanced nasopharyngeal carcinoma. Int J Radiat Oncol Biol Phys 1995;33(3):569-77.

48. Chua DT, et al. Long-term survival after cisplatin-based induction chemotherapy and radiotherapy for nasopharyngeal carcinoma: a pooled data analysis of two phase III trials. J Clin Oncol 2005;23(6):1118-24.

49. Ma J, et al. Results of a prospective randomized trial comparing neoadjuvant chemotherapy plus radiotherapy with radiotherapy alone in patients with locoregionally advanced nasopharyngeal carcinoma. J Clin Oncol 2001;19(5):1350-57. 